\title{
Neways: Cancer Network for Welfare Aging
}

\section{Neways: Cancer Network for Welfare Aging}

Francisco Luis PIMENTEL $\triangle^{1,2,3}$, Carlos OLIVEIRA ${ }^{4}$, Jorge SOARES ${ }^{5}$, Manuel VERÍSSIMO ${ }^{6}$

Acta Med Port 2016 Apr;29(4):235-236 - http://dx.doi.org/10.20344/amp.7617

Palavras-chave: Geriatria; Hematologia; Oncologia Médica; Portugal.

Keywords: Geriatrics; Hematology; Medical Oncology; Portugal.

Atualmente, assiste-se ao envelhecimento progressivo da população, sendo que o grupo etário com mais de 65 anos corresponde a $19,1 \%$ da população portuguesa. ${ }^{1}$ Mais de $50 \%$ de todas neoplasias ocorrem após os 65 anos e, cerca de $27 \%$, após os 75 anos. $^{2}$ Nas próximas duas décadas, com o aumento da esperança de vida, o peso da doença oncológica nos idosos vai naturalmente aumentar, constituindo uma preocupação de saúde pública.

As terapêuticas antineoplásicas instituídas estão longe de serem as ideais para o doente oncológico idoso. Os idosos em comparação com populações mais jovens geralmente têm mais comorbilidades, tomam mais medicamentos, têm mais efeitos adversos, mais interações medicamentosas, maior variabilidade do estado nutricional e do estado de saúde crónica subjacente que pode contribuir para maiores diferenças farmacocinéticas e farmacodinâmicas. Estes dados são escassos em doentes idosos, essencialmente devido à baixa inclusão destes doentes em ensaios clínicos. Apesar de mais de metade dos doentes diagnosticados com cancro serem idosos, proporcionalmente, apenas 20 a $40 \%$ integram ensaios clínicos de fase II e III, e os que são envolvidos, a grande maioria tem menos de 70 anos. ${ }^{3}$

Perante este contexto, cuidar dos idosos com cancro requer competências multidisciplinares, e de preferência, uma relação de trabalho entre oncologistas e geriatras. É crucial a formação em competências geriátricas gerais e em especificidades do cancro no idoso e, prioritariamente, compreender a importância da avaliação global, particularmente perante um doente idoso frágil. Os doentes idosos podem beneficiar de estratégias terapêuticas anti-neoplásicas numa extensão comparável à dos benefícios em doentes mais jovens, desde que sejam tomadas algumas precauções. Estas incluem a seleção dos doentes com base na esperança de vida e na potencial tolerância ao tratamento, que pode ser maximizado com recurso a terapêuticas de suporte de que é exemplo o uso profilático de fatores de crescimento hematopoiéticos para a quimio- terapia moderadamente tóxica, a adequação da primeira dose do tratamento à função renal, o diagnóstico precoce e tratamento atempado de efeitos adversos, a prevenção de anemia e a seleção de fármacos com perfil tóxico mais tolerável.

Para que o tratamento seja dirigido da forma mais adequada possível ao doente idoso, é necessária a existência de instrumentos que permitam diminuir a incerteza da estratégia terapêutica a implementar e consequente minimização do risco. Neste sentido, existem ferramentas de avaliação geriátrica, de que são exemplo o questionário "G8" e a "Abordagem Geriátrica Compreensiva" (AGC). Este último instrumento aplicado no doente idoso com cancro tem vindo a demonstrar benefícios, quer no aumento da sobrevivência, quer na melhoria da qualidade de vida. ${ }^{4}$ Porém, o nível da sua utilização por parte das equipas assistenciais é ainda muito baixo.

Os objetivos da estratégia terapêutica nesta população não se resumem apenas ao aumento da sobrevivência, devendo também incluir resultados em relação ao estado funcional (expresso na capacidade de cuidar de si próprio e manter-se física e mentalmente independente) e à qualidade de vida. ${ }^{3}$ Uma vez que o cancro provoca uma deterioração progressiva mais acentuada na autonomia do idoso, aspetos como a manutenção da independência, a prestação de cuidados de enfermagem e serviços sociais, ou o adequado suporte sintomático, revestem-se de um maior significado, sendo necessário uma abordagem de equipa interdisciplinar para avaliação e intervenção.

Esta tendência demográfica levou ao desenvolvimento por todo o mundo de programas dedicados à abordagem de doentes idosos com cancro. Diversas associações científicas internacionais têm prestado atenção a este tema, nomeadamente a Sociedade Internacional Geriatria Oncológica (International Society of Geriatric Oncology - SIOG), a Associação Europeia de Hematologia (European Hematology Association - EHA), a Sociedade Europeia de Oncologia (European Society for Medical Oncology - ESMO) e a

\footnotetext{
1. Lenitudes Medical Center \& Research. Santa Maria da Feira. Portugal.

2. Departamento Ciências Médicas. Universidade de Aveiro. Aveiro. Portugal.

3. Representante Nacional da "International Society of Geriatric Oncology". Geneva. Switzerland.

4. Liga Portuguesa contra o Cancro. Coimbra. Portugal.

5. Programa Gulbenkian Inovar em Saúde. Fundação Calouste Gulbenkian. Lisboa. Portugal.

6. Núcleo de Geriatria. Sociedade Portuguesa de Medicina Interna. Lisboa. Portugal.

$\square$ Autor correspondente: Francisco Luis Pimentel. f.pimentel@lenitudes.pt

Recebido: 14 de março de 2016 - Aceite: 05 de abril de 2016 | Copyright @ Ordem dos Médicos 2016
} 
Sociedade Americana de Hematologia (American Society of Hematology - ASH). Neste âmbito, estão a ser geradas evidências que suportam a necessidade de adaptação da prática médica nestas especialidades às características dos doentes em termos de idade, comorbilidades, estado nutricional, cognitivo ou psicológico. Dado que a idade por si só é um fraco descritor da heterogeneidade no processo de envelhecimento, torna-se fundamental determinar a idade funcional do doente, isto é, a idade atribuída de acordo com a sua capacidade funcional, e considerá-la nas decisões de tratamento, em detrimento da idade cronológica.

Apesar das diretivas de intervenção propostas pelas sociedades científicas, é ainda notória a falta de informação nesta área, nomeadamente:

- Dados robustos e evidence-based sobre a epidemiologia das neoplasias neste grupo etário e sobre a forma como os doentes são tratados; até aqui, o conhecimento adquirido tem sido apenas empírico, decorrente da experiência e prática clínica diárias;

- Inquéritos relativos à vontade dos doentes e ao que eles desejam, sobre a sua disposição para a realização de quimioterapia de baixa e alta toxicidade;

- Literacia das famílias dos doentes, juntamente com um investimento significativo na área do relacionamento intra-familiar - os cuidadores familiares desempenham um papel essencial na compreensão $e$ gestão da informação sobre a doença, sendo importante investigar especificamente os níveis de literacia dos cuidadores. ${ }^{5}$

- Pelos motivos mencionados, torna-se evidente a importância de debater estes assuntos a nível nacional, e verificar qual a necessidade de desenvolvimento de uma estratégia neste âmbito que permita delinear atividades visando a melhoria ou otimização da realidade portuguesa. É neste contexto que surge o projeto "Neways - Cancer Network for Welfare Aging", cujos objetivos principais são:

- Estimular a investigação no sentido de produzir conhecimento relativo à realidade oncogeriátrica Portuguesa e, em função do conhecimento produzido, gerar informação. Isto passa pela produção de dados epidemiológicos nacionais relativos ao doente idoso com neoplasias, e pela realização de análises de custo-benefício relativas ao seu tratamento e à gestão da sua doença;

\section{REFERÊNCIAS}

1. Instituto Nacional de Estatística. Censos 2011:Resultados provisórios. Lisboa: INE; 2011.

2. Registo Oncológico Regional Norte. Projeções de Incidência de Cancro Região Norte - 2013, 2015 e 2020. Porto: RORN; 2013

3. Jørgensen T, Hallas J, Land L, Herrstedt J. Comorbidity and polypharmacy in elderly cancer patients: The significance on treatment outcome and tolerance. J Geriatr Oncol. 2010;1:87-102.
- Desenvolver boas práticas para a prestação de cuidados de saúde ao doente idoso com doença oncológica, que se corporizem em recomendações nacionais tendo por base as normas internacionais já existentes;

- Promover o patient advocacy, isto é, a decisão participada do doente e cuidadores nos processos de decisão relativos à doença, nomeadamente na decisão da estratégia de tratamento mais adequada;

- Aumentar o awareness dos profissionais de saúde sobre as características especiais da subpopulação idosa, estimulando formação específica nesta área para médicos e profissionais de saúde;

- Aumentar a utilização de ferramentas que permitam avaliar os doentes oncológicos idosos em Portugal;

Promover a discussão e a responsabilidade da sociedade civil sobre a importância de um correto exercício da oncologia geriátrica.

O Steering Committee deste projeto é constituído pelos seguintes membros:

- Francisco Luis Pimentel - representante nacional da SIOG;

- Carlos Oliveira - Liga Portuguesa contra o Cancro;

- Jorge Soares - Fundação Calouste Gulbenkian;

- Manuel Veríssimo - Sociedade Portuguesa de Medicina Interna - núcleo de geriatria.

No âmbito do projeto serão definidos grupos de trabaIho correspondentes a áreas específicas, que procurarão identificar mitos e barreiras no contexto da oncologia geriátrica dentro das respetivas áreas de atuação. Mais concretamente, propôs-se a criação de quatro grupos principais: clínica; economia e políticas da saúde; investigação epidemiológica e social; outras áreas de atuação (literacia em saúde, tecnologias de informação para os doentes, questões éticas, patient advocacy e awareness).

Neste sentido, procura-se desenvolver uma network de especialistas que possa definir e apresentar uma estratégia que responda à necessidade de identificar os doentes que são suscetíveis de beneficiar e tolerar um tratamento agressivo, através da utilização de ferramentas de avaliação geriátrica. Pretende-se, ainda, propor ações que visem a discussão pela sociedade civil sobre a importância de melhorar a qualidade de vida do doente idoso oncológico.

4. Molina-Garrido MJ, Guillen-Ponce C, Sanchez Castellano C, Montero Errasquin B, Mora-Rufete A, Cruz-Jentoft AJ. Tools for decisionmaking in older cancer patients. Role of the comprehensive geriatric assessment. Anticancer Agents Med Chem. 2014;14:651-6.

5. Bevan JL, Pecchioni LL. Understanding the impact of family caregiver cancer literacy on patient health outcomes. Patient Educ Couns. 2008;71:356-64. 